\title{
Infrared Spectra in Polarized Light of Crystalline Chloroform
}

E. A. D'Alessio, E. Dodero, and C. Pomposiello

Citation: The Journal of Chemical Physics 57, 4136 (1972); doi: 10.1063/1.1678041

View online: https://doi.org/10.1063/1.1678041

View Table of Contents: http://aip.scitation.org/toc/jcp/57/10

Published by the American Institute of Physics

\section{PHYSICS TODAY}

ADVANCED LIGHT CURE ADHESIVES

READ NOW

Take a closer look at what these environmentally friendly adhesive systems can do
PRESENTED BY (8) MASTERBOND 
a.u., with binding energies of $0.063 \mathrm{eV}$ for $(4,1) s+$ $(4,1) p$ and $0.090 \mathrm{eV}$ for $5 s+5 p$. This $R_{e}$ and the tiny binding energies compare well with the elaborate $a b$ initio configuration interaction calculations of Browne, ${ }^{7}$ who used a mixed STO-elliptical coordinate basis set with some exponent optimization. He found $R_{e}=4.25$ from his simplest wavefunction, and calculated binding energies ranging from 0.089 to $0.117 \mathrm{eV}$ for different choices of trial wavefunctions. Rather conservative error estimates placed the estimated true binding energy in the $0.038-0.15 \mathrm{eV}$ range, probably nearer the upper end. Thus we conclude that our model potential approach has once again given a good representation of the energy of a many-electron system.

\section{SUMMARY AND CONCLUSIONS}

The one-electron molecular model potential scheme introduced in Sec. I has been applied in Sec. II to $\mathrm{Li}_{2}{ }^{+}, \mathrm{Na}_{2}{ }^{+}$, and $\mathrm{LiH}^{+}$. The behavior with increasing basis set flexibility and comparion with other more elaborate all-electron studies show that our approach is a convenient and accurate theory for such systems. Extensions of the method to polyatomic and manyvalence electron systems are now being made.

Note added in proof: We have also calculated potential curves for $\mathrm{Nali}^{+}$and $\mathrm{NaH}^{+}$. For $\mathrm{NaLi}^{+}$, we calculate $D_{e}=0.95 \mathrm{eV}$ at $R_{e}=6.4$ a.u. for the $5 s+5 p$ wavefunction and $0.91 \mathrm{eV}$ at 6.4 a.u. for the $(4,1) s+$ $(2,1) p$ wavefunction, in good agreement with the extended STO basis SCF MO result of $0.92 \mathrm{eV}$ at 6.5 a.u. [P. J. Bertoncini, G. Das, and A. C. Wahl, J. Chem. Phys. 52, $5112(1970)]$. The results for $\mathrm{NaH}^{+}$are $D_{e}=0.027 \mathrm{eV}$ at $R_{e}=5.4$ a.u. for $5 s+5 p$, and $0.022 \mathrm{eV}$ at 5.75 a.u. for $(4,1) s+(4,1) p$. Since we know of no other calculations on the $\mathrm{NaH}^{+}$system, these may be viewed in light of our other work as reasonably accurate predictions.

\footnotetext{
* The Radiation Laboratory is operated under contract with the U.S. Atomic Energy Commission. This is AEC Document No. COO-38-853. Partial support of this work has also come from the Petroleum Research Fund, administered by the American Chemical Society.

1 M. E. Schwartz and J. D. Switalski, J. Chem. Phys. 57, 4125 (1972), previous paper.

${ }^{2}$ Of course the $\mathrm{H}$ atom in $\mathrm{LiH}^{+}$has no core or $V_{M}$, and we will need other basis functions for $\mathrm{H}$, to be described later.

${ }^{3}$ J. T. J. Huang, M. E. Schwartz, and G. V. Pfeiffer, J. Chem. Phys. 56, 755 (1972).

${ }^{4}$ We use atomic units: energy, 1 a.u. $=27.21 \mathrm{eV}$; length, 1 a.u. $=$ $0.52917 \AA$. This $\mathrm{Li} 2 s$ reference energy is for the $5 \mathrm{G}$ calculation (Table I of I).

${ }^{5}$ W. J. Hehre, R. F. Stewart, and J. A. Pople, J. Chem. Phys. 51,2657 (1969).

${ }^{6}$ The exact $\mathrm{H}$ ground state energy is -0.5 a.u. This value is that due to the $5 s$ basis for $\mathrm{H}$.

${ }^{7}$ J. C. Browne, J. Chem. Phys. 41, 3495 (1964).
}

\title{
Infrared Spectra in Polarized Light of Crystalline Chloroform
}

\author{
E. A. D'Alessio, * E. Dodero, and C. Pomposiello $\dagger$ \\ Comision Nacional de Energia Atomica, Avenida del Libertador 8250, Buenos Aires, Argentina
}

(Received 8 January 1971)

\begin{abstract}
High resolution spectra in polarized light of oriented crystalline films of chloroform at $77^{\circ} \mathrm{K}$ were obtained. The ratio of intensities of Davydov components for different angles of polarization were also determined. Comparison with the oriented gas model approximation allowed the assignment of components to the proper representations of the $D_{2 h}$ factor group: all bands were assigned to the infrared active factor group symmetry species $B_{1 u}, B_{2 u}$, and $B_{3 u}$ corresponding to vibrations along $\mathbf{a}, \mathbf{b}$, and crystallographic axes. Comparison with results of measurements in solid solutions in argon made by King allowed the distinction between crystallographic effect and isotopic shifts.
\end{abstract}

\section{INTRODUCTION}

The calculation of intermolecular forces in molecular crystals requires a detailed knowledge of crystal structure and a correct assignment of Davydov components to the symmetry species of the space group. At the present time only a few molecules have been studied in the crystal phase and a complete correlation of crystal and molecular bands has been made. Recently Marzocchi et al. ${ }^{1}$ have succeeded in the assignment of Davydov components in crystalline benzene and reasonably good agreement with theoretical calculations ${ }^{2}$ were obtained.

In this paper we present experimental results on infrared spectra in polarized light of crystalline chloroform and an assignment of its Davydov components is made. Calculations of the intermolecular field will be presented at a later time.

Several years ago, Lisitsa and Tsyashchenko ${ }^{3}$ measured the dependence of vibrational frequencies with temperature in solid chloroform.

Owing to the low resolution they had at that time and 
to the unrealistic model of two molecules per unit cell which they used in their calculations, this work is of little help in the assignment of crystal modes.

More recently, King ${ }^{4}$ measured infrared spectra for chloroform and other substances in solid argon matrices at $20^{\circ} \mathrm{K}$; he also made an intramolecular force field calculation, with parameters transferred from other related molecules. ${ }^{5}$ King's experimental results are very helpful because he determined very accurately the isotopic shifts due to the presence of ${ }^{35} \mathrm{Cl}$ and ${ }^{37} \mathrm{Cl}$ atoms and allow us to separate this effect from crystalline phenomena, as both are of the same order of magnitude.

Kimoto and Yamada ${ }^{6}$ measured absolute intensities in solid chloroform and found crystal splitting in some of the vibrational bands.

In 1966, Fourme and Renaud ${ }^{7}$ determined the crystal structure of chloroform at $185^{\circ} \mathrm{K}$ by x-ray diffraction. Their results-although erroneous in the estimation of the position of hydrogen atoms-show that chloroform crystallizes in the orthorhombic system, space group Pnma $\left(D_{2 h}{ }^{16}\right)$ with four molecules per unit cell.

Our assignment of the vibrations of crystalline chloroform will be based on this structure, with our modification on the position of the hydrogen atoms.

\section{EXPERIMENTAL}

Reagent grade chloroform was distilled under vacuum in absence of light, dried with calcium chloride and kept frozen between experiments. The purity was controlled by gas chromatography in a Varian Aerograph, model 1520 equipment; less than 10 parts per million of carbon tetrachloride and ethyl alcohol were found in the final product.

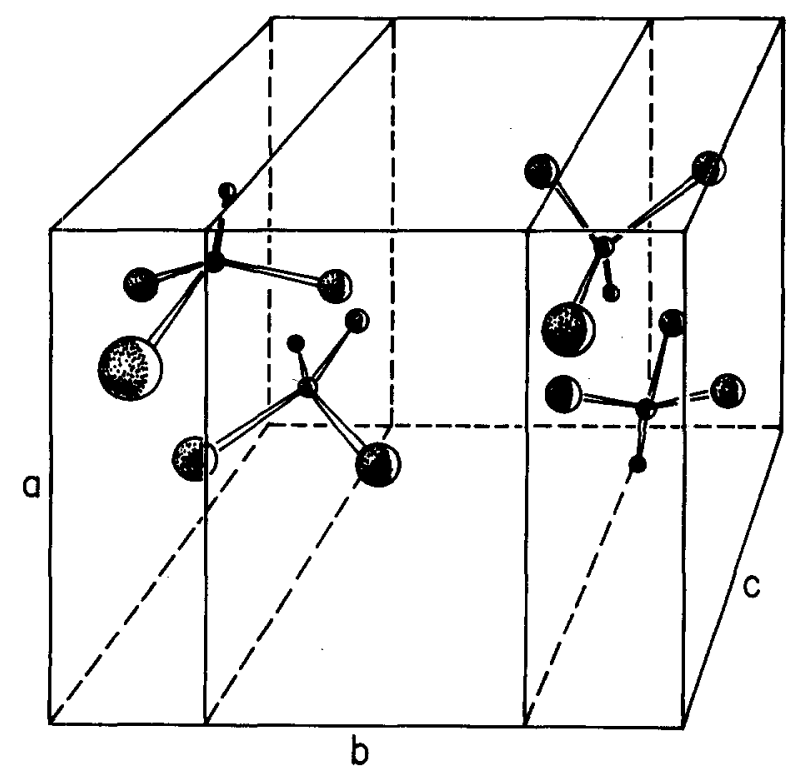

FIG. 1. Crystal structure of solid chloroform, orthorhombic space group Pnma $\left(D_{2 h^{16}}\right)$.

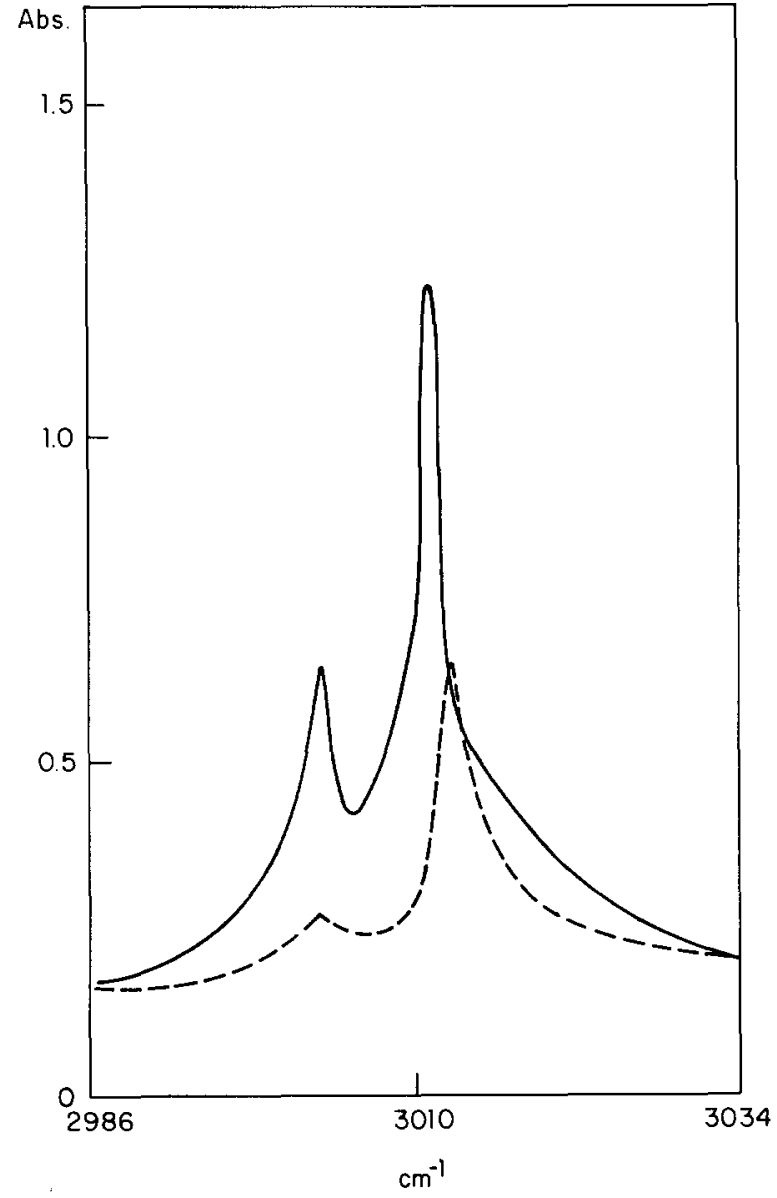

FIG. 2. $\nu_{1}\left(A_{1}\right)$ vibration of solid chloroform. Full line: $\alpha$ polarized; dotted line: $\beta$ polarized.

Single crystals and oriented polycrystals were grown starting from liquid layers enclosed between two CsI windows in a conventional cryostat. The enhancement of orientation effects was obtained by means of a temperature gradient along the liquid layer. The cell was kept with an overpressure of dry air while the growth was performed and maintained under vacuum during experiments. This procedure avoided the use of careful vacuum seal in the growth chamber.

The intensity of spectra was controlled by the use of different spacers; no spacer was used to record the strongest peak $\left(767 \mathrm{~cm}^{-1}\right)$.

With this technique, samples of $2 \mathrm{~cm}^{2}$ showing complete extinction between crossed polarizers were easily obtained.

The crystal thickness was measured by interference and by calibration with several bands of liquid $\mathrm{CCl}_{3} \mathrm{H}$ against a variable-path calibrated cell.

The infrared spectra between 700 and $4000 \mathrm{~cm}^{-1}$ were recorded with a Perkin-Elmer, model 225 spectrophotometer; spectra between 200 and $780 \mathrm{~cm}^{-1}$ were obtained in a Beckman, model IR 11 instrument. 
TABLE I. Correlation between molecular, site, and factor groups for crystalline chloroform, and activity in the crystal.

\begin{tabular}{|c|c|c|c|}
\hline $\begin{array}{l}\text { Molecular } \\
\text { group }\end{array}$ & $\begin{array}{l}\text { Site } \\
\text { group }\end{array}$ & $\begin{array}{l}\text { Factor } \\
\text { group }\end{array}$ & Activity \\
\hline \multirow[t]{5}{*}{$\mathrm{C}_{3 \mathrm{v}}$} & $\bar{C}_{s(x)}$ & $\mathrm{D}_{2 \mathrm{~h}}$ & \\
\hline & & $-A_{g}$ & $R^{a}$ \\
\hline & & $\Gamma^{-B_{1 g}}$ & $R$ \\
\hline & & $+B_{2 g}$ & $\mathrm{R}$ \\
\hline & & $B_{3 g}$ & $R$ \\
\hline \multirow{3}{*}{$\mathrm{A}_{2}$} & & & $\mathbb{R}^{b}$ \\
\hline & & L_B2u(y) & $\mathbb{R}$ \\
\hline & & _ $B 3 u(x)$ & $\mathbb{R}$ \\
\hline
\end{tabular}

Raman active in the crystal.

b Infrared active in the crystal.
The resolution was better than $0.5 \mathrm{~cm}^{-1}$ in the whole range.

The temperature was measured with a copper-constantan thermocouple pressed between the windows and controlled with a heater powered with a variable ac source. Temperature stability was better than $2^{\circ} \mathrm{C}$.

Nonoriented samples were obtained by freezing gas sprayed over a CsI window, kept at liquid nitrogen temperature. The crystallization is observed only after repeated annealing of the specimens at temperatures between 10 and $30^{\circ} \mathrm{C}$ below the freezing point $\left(-60^{\circ} \mathrm{C}\right)$.

Scannings of crystal samples at different temperatures between 77 and $185^{\circ} \mathrm{K}$ do not show significative changes after annealing.

\section{RESULTS AND DISCUSSION}

It was said that crystalline chloroform belongs to the orthorhombic Pnma $\left(D_{2 h}{ }^{16}\right)$ space group, with four molecules per unit cell, located at sites of $C_{s}$ symmetry (see Fig. 1).

The correlation (Table I) between the molecular

TABLE II. Infrared spectrum of chloroform. Frequencies, assignments, polarization, and integrated intensities in the crystal.

\begin{tabular}{|c|c|c|c|c|c|}
\hline \multirow{2}{*}{$\begin{array}{l}\text { Molecular } \\
\text { mode }\end{array}$} & \multicolumn{2}{|c|}{ Observed frequencies $\left(\mathrm{cm}^{-1}\right)$} & \multirow[b]{2}{*}{ Polarization } & \multirow{2}{*}{$\begin{array}{l}\text { Integrated } \\
\text { intensities }^{b}\end{array}$} & \multirow{2}{*}{$\begin{array}{l}\text { Crystal } \\
\text { modes }\end{array}$} \\
\hline & Liquid $^{\mathfrak{a}}$ & Crystal & & & \\
\hline \multirow{2}{*}{$\boldsymbol{\nu}_{1}\left(A_{1}\right)$} & 3019 & 3013 & $\beta$ & 0.15 & $B_{3 u}$ \\
\hline & & 3012 & $\alpha$ & 1.00 & $B_{1 u}$ \\
\hline$\nu_{4}+\nu_{2}(?)$ & 3003 & 3003 & & & \\
\hline \multirow[t]{2}{*}{$\nu_{2}\left(A_{1}\right)$} & 668 & 672 & $\alpha$ & 1.00 & $B_{14}$ \\
\hline & & 670.5 & $\beta$ & 0.21 & $B_{3 u}$ \\
\hline \multirow[t]{6}{*}{$\boldsymbol{\nu}_{3}\left(A_{1}\right)$} & 366 & 369.8 & $\beta$ & 0.74 & $B_{3 u}\left(\mathrm{C}^{36} \mathrm{Cl}_{3} \mathrm{H}\right)$ \\
\hline & & 369.5 & $\alpha$ & 1.00 & $B_{1 u}\left(\mathrm{C}^{35} \mathrm{Cl}_{3} \mathrm{H}\right)$ \\
\hline & & 367.3 & $\beta$ & & $B_{3 u}\left(\mathrm{C}^{36} \mathrm{Cl}_{2}{ }^{37} \mathrm{ClH}\right)$ \\
\hline & & 366.7 & $\alpha$ & & $B_{1 u}\left(\mathrm{C}^{35} \mathrm{Cl}_{2}^{37} \mathrm{ClH}\right)$ \\
\hline & & 364.2 & $\beta$ & & $B_{3 u}\left(\mathrm{C}^{36} \mathrm{Cl}^{37} \mathrm{Cl}_{2} \mathrm{H}\right)$ \\
\hline & & 363.8 & $\alpha$ & & $B_{1 u}\left(\mathrm{C}^{35} \mathrm{Cl}^{37} \mathrm{Cl}_{2} \mathrm{H}\right)$ \\
\hline \multirow[t]{3}{*}{$\nu_{4}(E)$} & 1213 & 1223 & $\beta$ & 1.00 & $B_{3 u}$ \\
\hline & & 1207 & $\alpha$ & 0.20 & $B_{1 u}$ \\
\hline & & 1203 & $\beta$ & .. & $B_{2 u}$ \\
\hline \multirow[t]{3}{*}{$\nu_{5}(E)$} & 759 & 767 & $\alpha$ & 0.20 & $B_{1 u}$ \\
\hline & & 750 & $\boldsymbol{\beta}$ & 1.00 & $B_{3 u}$ \\
\hline & & 737 & $\beta$ & $\cdots$ & $B_{2 u}$ \\
\hline \multirow[t]{3}{*}{$\nu_{6}(E)$} & 252 & 271 & $\alpha$ & $\ldots$ & $B_{1 u}$ \\
\hline & & 269 & $\beta$ & $\cdots$ & $B_{3 u}(?)$ \\
\hline & & 263 & $\beta$ & $\ldots$ & $B_{2 u}(?)$ \\
\hline
\end{tabular}

$\nu_{1}, \nu_{2}, \nu_{4}$, and $\nu_{5}$, measured by M. P. Lisitsa and Tsyashchenko; $\nu_{3}$ and $\nu_{6}$ measured in this work.

b For comparison purposes with the oriented gas model, the intensity is defined relative to the strongest component in each multiplet. The values are corrected for selective polarization of the spectrometer, and measured in oriented crystals. The accuracy of these experimental results is within $5 \%-10 \%$. 
group $\left(C_{3 v}\right)$, the site group $\left(C_{8}\right)$ and the factor group $\left(D_{2 h}\right)$ shows that each band which belongs to the $A_{1}$ symmetry species in the chloroform molecule should split, in the crystal, into two infrared active components, with symmetries $B_{1 u}$ and $B_{3 u}$, polarized along the $c$ and a crystal axes, respectively. The table also shows that bands belonging to the $E$ representation in the molecule split into two components-with symmetries $A^{\prime}$ and $A^{\prime \prime}$-when the molecule occupies a $C_{s}$

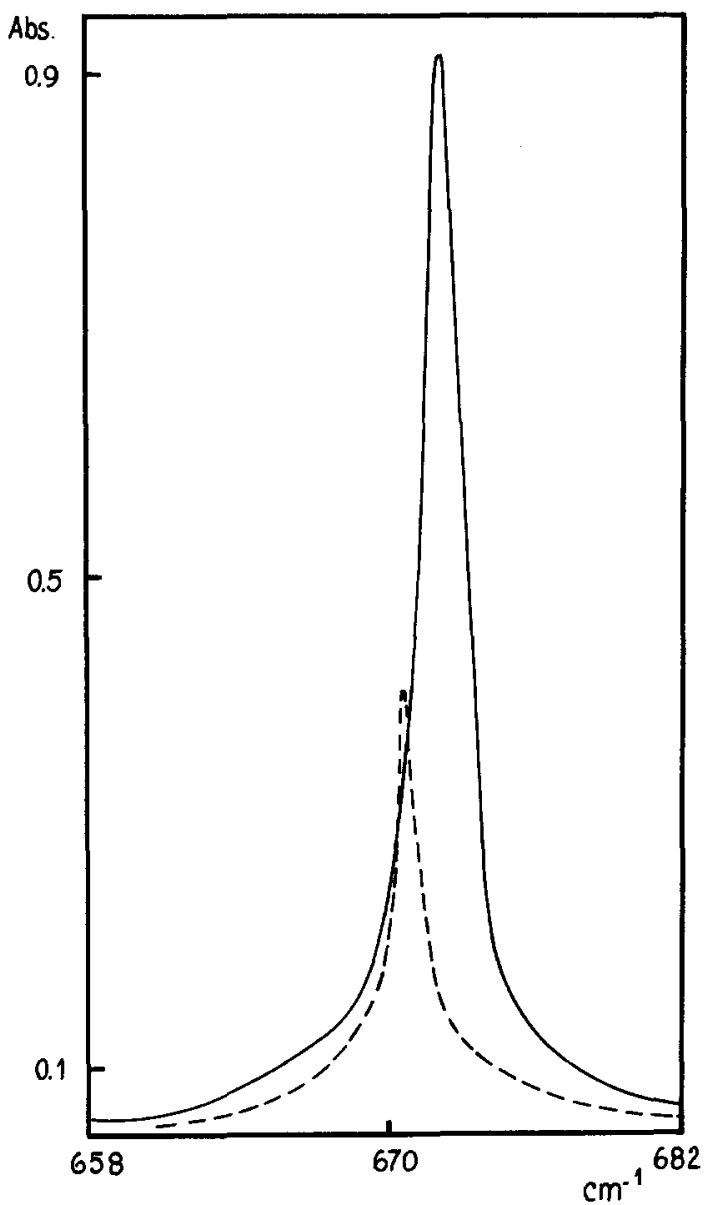

FIG. 3. $\nu_{2}\left(A_{1}\right)$ vibration of solid chloroform. Full line: $\alpha$ polarized; dotted line: $\beta$ polarized.

site in the crystal. Each one of these bands will have another splitting-the factor group splitting-into two components: $A^{\prime}$ in $B_{1 u}$ and $B_{3 u}$ infrared active, and $A^{\prime \prime}$ in $A_{u}$, infrared inactive and $B_{2 u}$, infrared active. $B_{1 u}$ and $B_{3 u}$ will be polarized along $\mathrm{C}$ and $\mathrm{a}$ axes and $B_{2 u}$ along the b crystal axis.

It should be mentioned that, as can be seen in Table I, there are also several Raman active components. These are not studied at the present time.

It is quite apparent that the study in polarized light will allow us to classify the bands in three groups accord-

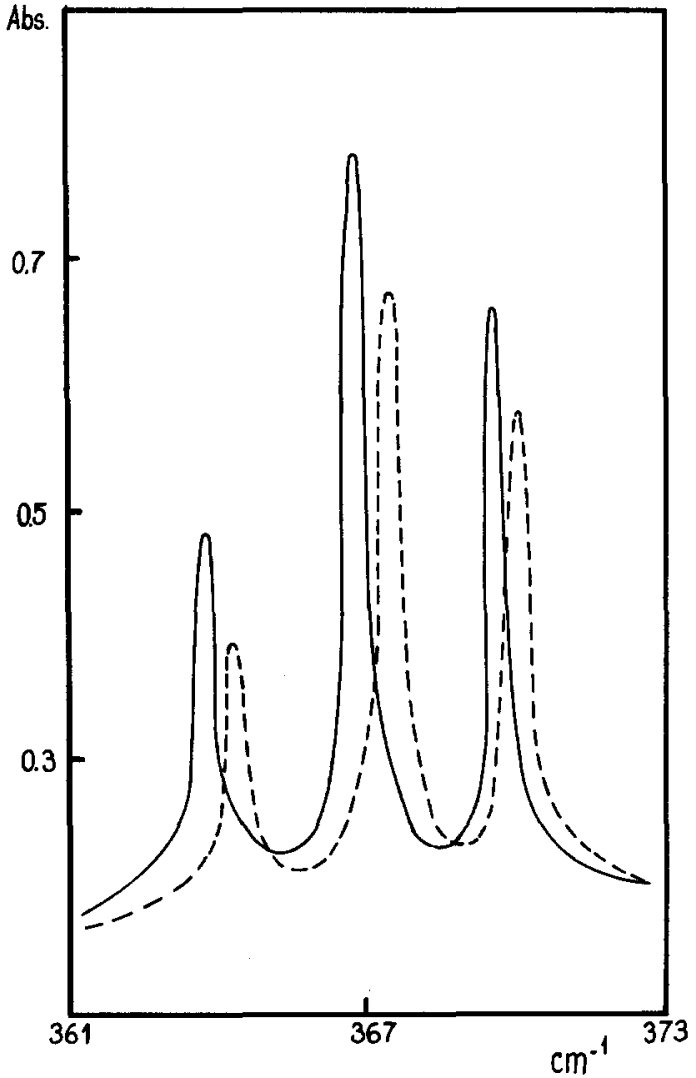

FIG. 4. $\nu_{3}\left(A_{1}\right)$ vibration of solid chloroform. Full line: $\alpha$ polarized; dotted line: $\beta$ polarized.

ing to their symmetries, or according to their directions of vibration; provided that the crystal is irradiated with a parallel beam along each crystal axis.

This last requirement is not fulfilled in general because not necessarily a (100), (010) or (001) face will grow against the CsI substrate. In general an arbitrary ( $h k l$ ) face will be observed.

Our spectra of crystalline chloroform in polarized light (Figs. 2-7) show that every band is totally polarized, either along the direction of the temperature gradient or perpendicular to it ( $\alpha$ and $\beta$ directions, respectively).

The following remarks can be made: (i) the crystal

TABLE III. Oriented gas model approximation.

\begin{tabular}{cccc}
\hline \hline \multirow{2}{*}{$\begin{array}{c}\text { Molecular } \\
\text { mode }\end{array}$} & $B_{1 u}(z)$ & $B_{2 u}(y)$ & $B_{3 u}(x)$ \\
\cline { 2 - 4 }$A_{1}$ & 1.00 & $\ldots$ & 0.13 \\
$E$ & 0.13 & $1.13^{\mathrm{a}}$ & 1.00 \\
\hline
\end{tabular}

- For comparison purposes, the intensity of $B_{3 u}(x)$ is taken as unity. 


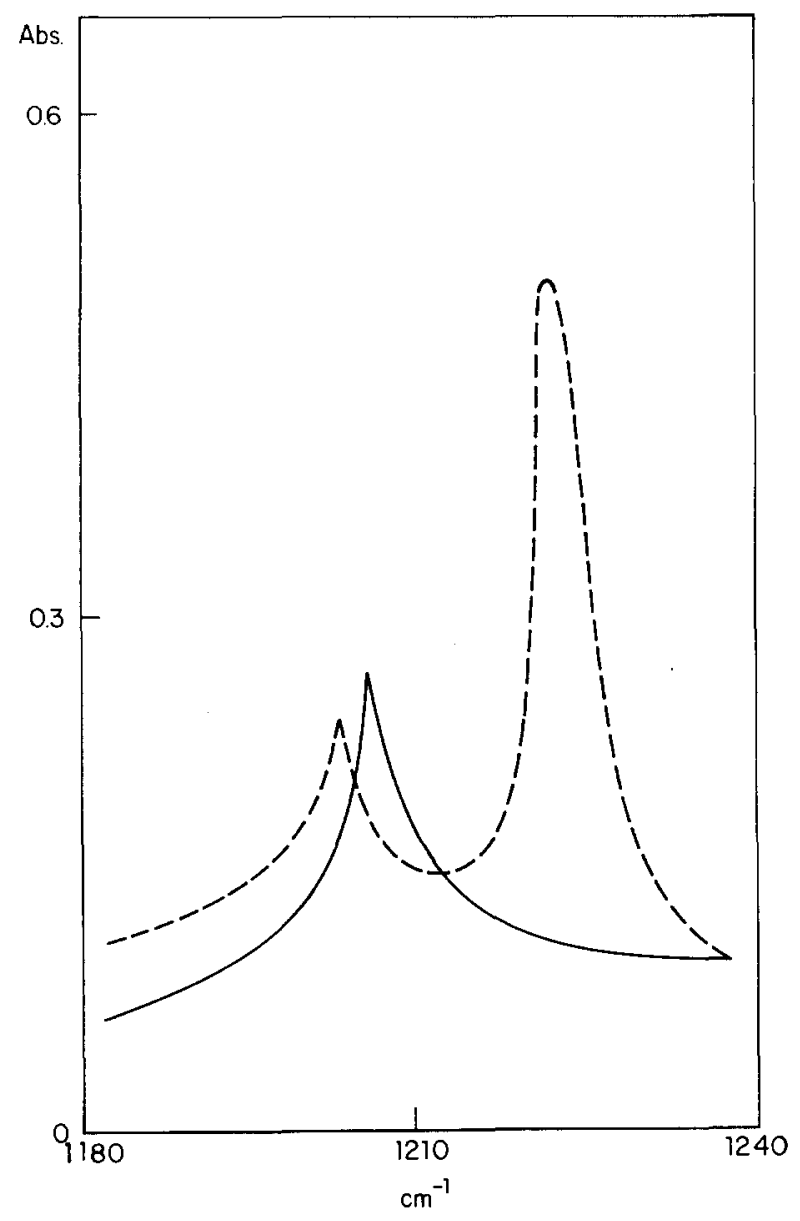

I'IG. 5. $\nu_{4}(E)$ vibration of solid chloroform. Full line: $\alpha$ polarized; dotted line: $\beta$ polarized.

is totally ordered (experimental error: less than $2 \%$ ); (ii) one definite face, and always the same, is growing against the CsI substrate. Statement (ii) is confirmed by the observation of a constant ratio of intensities for the two perpendicular directions in every observed crystalline specimen.

In Table II we have collected the infrared bands observed in the crystal together with their assignment and frequencies in the liquid, their polarization, relative integrated intensities, and tentative assignment in a crystal factor group $D_{2 h}$.

The relative intensities of Davydov components for oriented crystals corresponding to one band in the gas molecule depends on several factors: (i) intrinsic intensities; (ii) reflections in the crystal surface; (iii) angle between each direction of vibration and the electric vector incident to the sample; (iv) degree of polarization produced by the spectrometer.

Factor (iv) is known for the instruments and corrections are applied for each band and angle of polarization.

Factor (ii) is small for the CsI-chloroform contact and the losses do not exceed $2 \%{ }^{8}$ The selective reflec- tion for different polarizations must be well below that figure. The intrinsic intensities should be independent in first approximation on the state of orientation of the sample. This means that the differences between oriented polycrystalline samples and disordered specimens should be attributed to factor (iii) and an estimate of the orientation must be obtained.

The oriented gas model ${ }^{9}$ is a zeroth-order approximation to predict the relative intensities for the Davydov components in a given multiplet. It is useful to have an order of magnitude which sometimes is enough to assign the components to the proper symmetry species. In Table III we present the calculations for predicted intensities of bands belonging to the $C_{3 n}$ group projected on the crystal axes of the Pnma space group.

Bands in the molecule that belong to the $A_{1}$ species split into two components in the crystal and those belonging to the $E$ species split into three components, in total agreement with the predicted correlation. Two components of each $E$ vibration are $\beta$ polarized and one is $\alpha$ polarized, thus one crystal axis must be coincident or almost coincident with the $\alpha$ direction. The more intense component of each $A_{1}$ vibration (which, from Table III, must be the $B_{1 u}$ component) is $\alpha$ polarized, thus, it is the crystal axis which is coincident or almost coincident with $\alpha$.

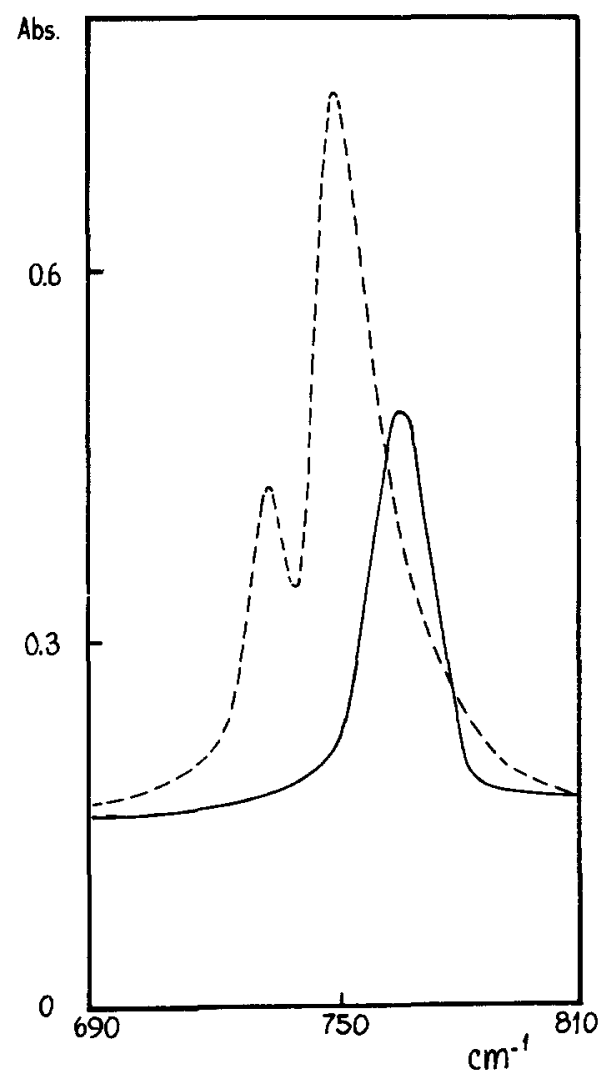

FIG. $6 . \nu_{5}(E)$ vibration of solid chloroform. Full line: $\alpha$ polarized; dotted line: $\beta$ polarized. 
One of the $\beta$ polarized $E$ components diminishes markedly in intensity in the single crystal spectra, compared to the polycrystalline sample; while the $\beta$ polarized $A_{1}$ component $\left(B_{3 u}\right)$ and the remaining $\beta$ polarized $E$ component do not (Fig. 8 and 9 ). Thus the stronger (in the single crystal spectra) $\beta$ polarized $E$ component must belong to the $B_{3 u}$ species, and the weaker component to the $B_{2 u}$ species, with the $\mathbf{b}$ axis roughly perpendicular to the CsI window.

In $\nu_{1}$, the ratio of integrated intensities for the two observed components is about $1 / 0.15$; since this ratio is close to the predicted value $\left(1 / 0.13\right.$ for $\left.I_{\alpha} / I_{\beta}\right)$ and the splitting is only $1 \mathrm{~cm}^{-1}$ we conclude that the $\mathrm{C}-\mathrm{H}$ stretching mode is very weakly perturbed by the crystal field.

The $\nu_{3}\left(A_{1}\right)$ band has a factor splitting of about 0.5 $\mathrm{cm}^{-1}$ whereas the isotopic shift is 2 to $3 \mathrm{~cm}^{-1}$. Three isotopic components are resolved and the factor splitting determined with its polarization. The intensity ratio $(1 / 0.74)$ is not close to $1 / 0.13$, but the measurement is erratic since the slit-width is greater than the true band half-width measured at $77^{\circ} \mathrm{K}$ in solid solutions.

The $\nu_{6}$ band (Fig. 7) is studied only in partially polarized light. Spectra are obtained by rotation of samples and profiting from the polarization produced

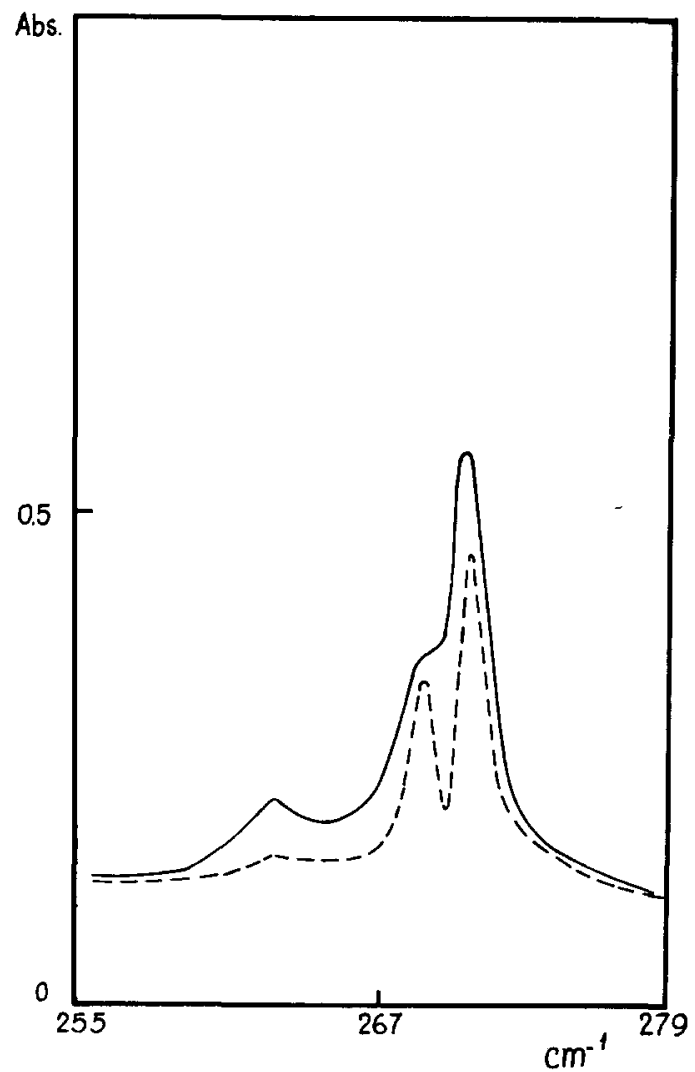

FIG. 7. $\nu_{6}(E)$ vibration of solid chloroform. Full and dotted lines: two positions of partial polarization.

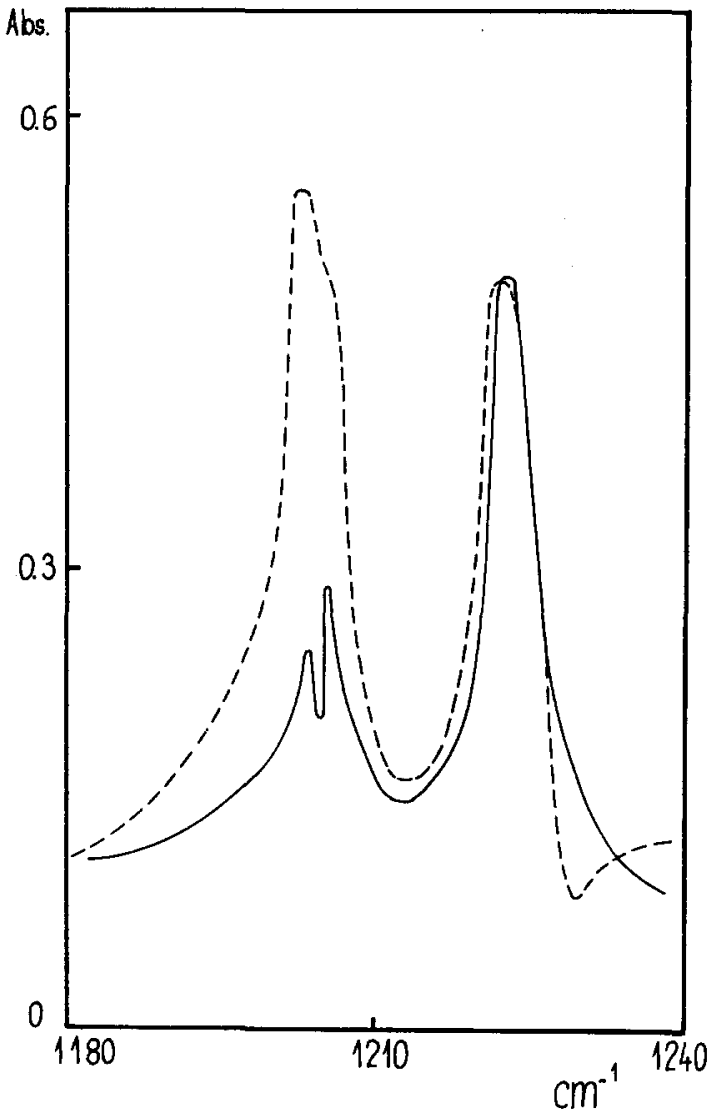

FIG. 8. $\nu_{4}$ vibration in oriented and nonoriented samples. Full line: oriented crystal; dotted line: nonoriented polycrystal.

by the spectrometer. It is thus possible to observe characteristics similar to those found in $\nu_{4}$ and $\nu_{5}$. The assignment of its components at $263 \mathrm{~cm}^{-1}$ and $269 \mathrm{~cm}^{-1}$ remain undetermined. Intensity measurements are not conclusive because of the lack of total polarization. Nevertheless, relative intensities in nonoriented samples favor the assignments of $269 \mathrm{~cm}^{-1}$ as a $B_{3 u}$ mode and $263 \mathrm{~cm}^{-1}$ as $B_{2 u}$ mode.

Four different isotopic molecules are present in the chloroform crystal: $\mathrm{C}^{35} \mathrm{Cl}{ }_{3} \mathrm{H}(49 \%), \mathrm{C}^{35} \mathrm{Cl}_{2}{ }^{37} \mathrm{ClH}(41 \%)$, $\mathrm{C}^{35} \mathrm{Cl}^{37} \mathrm{Cl}_{2} \mathrm{H}(9 \%)$, and $\mathrm{C}^{37} \mathrm{Cl}_{3} \mathrm{H}(1 \%)$; the first and fourth having $C_{3 v}$ symmetry and the second and third $C_{s}$ symmetry. The molecules with lower symmetry will have two possible orientations: one with the unequal chlorine atom on the symmetry plane $\left(C_{\delta}\right.$ site symmetry) and other with the unequal atom outside it ( $C_{1}$ site symmetry), giving rise to additional splittings. Nevertheless the distortions in the normal mode are so small (only $2 \%$ of change in mass and $0.6 \%$ in frequency) that the new components must be too weak to be observed and spectra for all four types of molecules must be very similar.

Only in $\nu_{3}\left(A_{1}\right)$ isotopic shifts are not masked by large half-widths; in that band no extra peaks are observed 


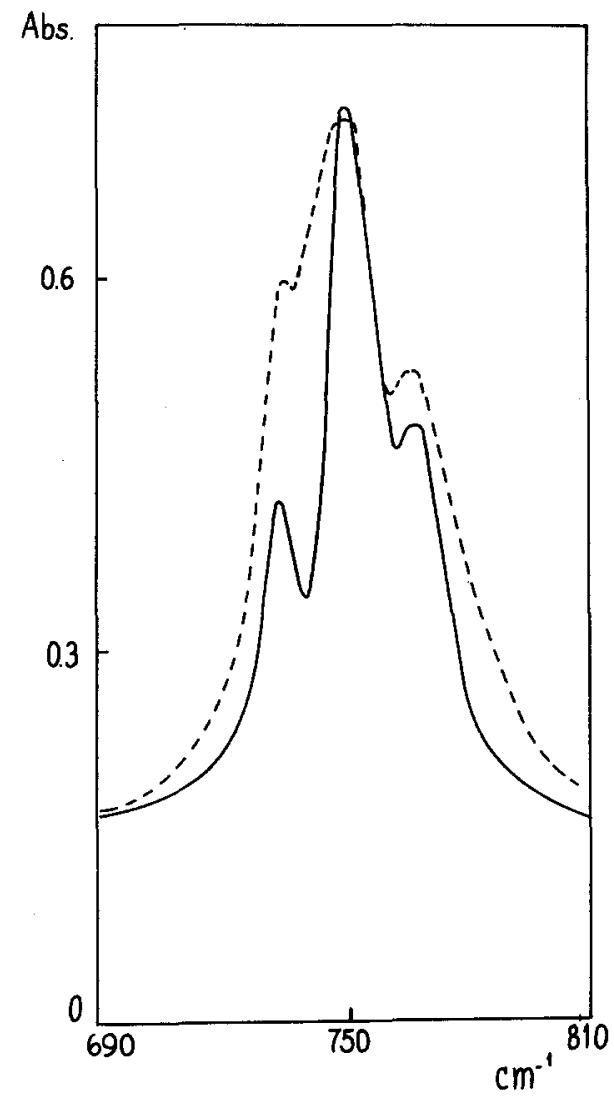

FIG. 9. $\nu_{5}$ vibration in oriented and nonoriented samples. Full line: oriented crystal; dotted line: nonoriented polycrystal.

and splittings are as if all four types of molecules had $C_{3 v}$ symmetry.

\section{CONCLUSION}

New information on the structure of crystalline chloroform is obtained by means of infrared spectroscopy in the present work. Band analysis and polarized light prove to be a powerful tool to analyze spectra of solid samples, allowing the classification of normal modes according to their directions of vibration and also helping to resolve Davydov components when the splitting are the order of magnitude of the experimental bands half-widths.

Although we used the oriented gas model approximation only to estimate the order of magnitude of intensity ratios, we found a surprisingly good agreement between experimental and theoretical values for some normal modes. This agreement indicates that intermolecular forces which build up the crystal are weak, as it can be corroborated from other evidences: (i) the largest frequency shift in the transition from gas to solid is only $8 \mathrm{~cm}^{-1}$; (ii) the total integrated intensity increases in $2 \%,{ }^{6}$ well within the experimental error, (iii) structural data show no measurable departure from tetrahedral shape in each individual molecule.

The assignment of each Davydov component to its proper symmetry species is of value for future intermolecular field calculations.

\section{ACKNOWLEDGMENTS}

The authors wish to thank Dr. Gerardo Videla and Mr. J. S. Orecchia for all their help and CITEFA for the use of the Perkin-Elmer 225 instrument.

* Member of Consejo Nacional de Investigaciones Cientificas y Tecnicas.

$\dagger$ Facultad de Ciencias Exactas y Naturales, Universidad de Buenos Aires.

1 M. P. Marzocchi, H. Bonadeo, and G. Taddei, J. Chem. Phys. $53,867(1970)$.

2 I. Harada and T. Shimanouchi, J. Chem. Phys. 44, 2016 (1966). N. Rich and D. A. Dows, Mol. Cryst. 5, 111 (1968).

${ }^{3}$ M. P. Lisitsa and Yu. P. Tsyashchenko; Opt. Spectrosk. 10, 157 (1961) [Opt. Spectrosc. 10, 79 (1961)].

${ }^{4}$ S. T. King, J. Chem. Phys. 49, 1321 (1968).

5 T. Shimanouchi, Pure Appl. Chem. 7, 131 (1963).

${ }^{6}$ A. Kimoto and H. Yamada, Bull. Chem. Soc. Japan 40, 243 (1967)

${ }^{7}$ R. Fourme and M. Renaud, Compt. Rend. 263, 69 (1966).

${ }^{8}$ M. P. Lisitsa and Yu. P. Tsyashchenko, Opt. Spectrosk. 6, 605 (1959) [Opt. Spectrosc. 6, 396 (1959)].

${ }^{\circ} \mathrm{G}$. C. Pimentel and A. L. Mc. Clellan, J. Chem. Phys. 20, 270 (1952). E. J. Ambrose, A. Elliot, and R. B. Temple, Proc. Roy. Soc. (London) A206, 192 (1951). 